\title{
Wall-Stability Analysis of a Climbing Robot
}

Hu BinLiang ${ }^{1, a}$, Chen GuoLiang ${ }^{2, ~ b}$, Chen GuangCheng ${ }^{2, c}$

\author{
${ }^{1}$ School of Mechanical and Electronic Engineering, Hunan University of Science and Technology, \\ Xiangtan 411201, Hunan, China \\ ${ }^{2}$ School of Mechanical and Electronic Engineering, Wuhan University of Technology, Wuhan, \\ 430070, China \\ ${ }^{2}$ School of Mechanical and Electronic Engineering, Wuhan University of Technology, Wuhan, \\ 430070, China

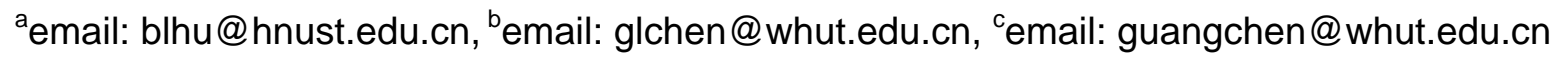

Keywords: Wall-climbing Robot; Permanent Magnet Adsorption; Stability Analysis

\begin{abstract}
A climbing robot's moving mechanism based on permanent magnet adsorption is proposed, which has a four-wheel structure including two driving permanent magnet wheels and two universal wheels. The problems of the robot's adsorption reliability is put a special effort to study. The force situations of robot at the horizontal position and vertical position are analyzed respectively, and the robot's mechanical model and the conditions of stability are built. The simulation experiments are carried out SolidWorks and ADAMS, and the results of simulations show its correctness.
\end{abstract}

\section{Introduction}

In the early 1960s, Japanese scholar successfully developed a robot capable of moving on the wall based on negative pressure. Since then, climbing robots become an important branch in robotics fields, and attracts the attention of many research institutions and scholars. A variety of climbing robot based on different principles are developed and used in nuclear industry, construction industry, shipbuilding and other [1][2][3].

For wall-climbing robot detecting the shaft-type pipe or the wall of pressure vessel, permanent magnet adsorption are widely adopted as adsorption mechanism because of its advantage of reliable adsorption and convenient implementation. At present, wall-climbing robot based on permanent magnet adsorption has two mainly types of mobile structure. They are crawler type embedded with rectangular permanent magnet and wheel type of ontology abdominal installation of permanent magnet. Wall-climbing robot of crawler type can adapt to different shape surface because of its large contact area, but the robot executes turns difficultly and overturn easily because of its large quality and scattered magnetic adsorption force. Wall-climbing robot of wheel type has a clearance with walls. But, its adsorption force is unstable and will dramatic change with the clearance of tiny changing. As can be seen, adsorbing of high reliability, walking of high flexibility and light weight are what wall-climbing robot needs to focus on solving [4] [5] [6].

A kind of wall-climbing robot based on the permanent magnetic wheel is developed to detect the inner wall of the cylindrical shaft with dense and complex structure. This paper focuses on setting the stability conditions that assure adsorption reliability and movement agility of the wheel robot by analyzing with mechanics. The model of the climbing robot is established by SolidWorks and run by ADAMS to verify the correctness of these stability conditions.

\section{The structure of moving mechanism}

The wall-climbing robot is employed to cylindrical shaft with the ontology structure material of $18 \mathrm{MnD} 5$ which has a good magnetic conductivity. So, the robot adopts the permanent magnetic adsorption method. The robot ues the moving mechanism of wheel, which adopts the four-wheel structure and two-wheel drive mode for the symmetrical balance of the robot's adsorption force. The 
wheel structure is shown in the Fig. 1. Two drive wheels employ the method of permanent magnet wheel and adopt the structure of front-and-rear layout, which can stagger the internal deceleration motor for reducing the transverse size of the robot. Two auxiliary wheels are symmetrically arranged along the center line of the robot, which are used to support the robot.

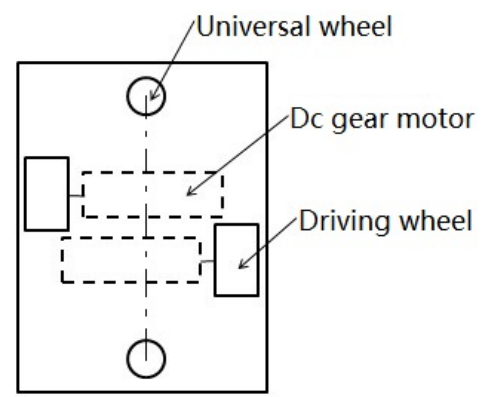

Fig. 1 Schematic diagram of moving mechanism

\section{Stability analysis of the moving mechanism}

The robot relies on the adsorption force from permanent magnet wheel to counteract the overturn couple acting upon its body and prevent itself slipping from the wall. However, the large adsorption force will sacrifice the movement flexibility robot, and cause the robot unable to move. Therefore, it is necessary to analyze the force of the robot on the wall. This paper analyzes robot's two extreme cases of the horizontal and vertical adsorption on the wall of the shaft.

Under the conditions of lateral adsorption and static, the robot's stress state is shown in Fig. 2. Fig. 2(a) is the force analysis of robot parallel to the wall's plane, and Fig. 2(b) is the force analysis of robot perpendicular to the wall and parallel to the direction of gravity.

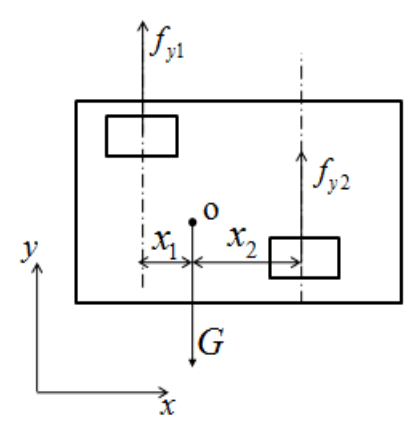

(a) Horizontal direction

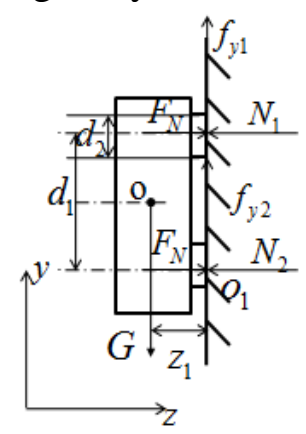

(b) Vertical direction

Fig. 2 Forces under the condition of robot's lateral static

In Fig. 2, $o$ is the center of gravity, $x_{1}$ and $x_{2}$ are the distance between the robot's center of gravity and two wheels' center respectively, $d_{1}$ is the distance of two wheels, $d_{2}$ is the width of two wheels, $z$ is the distance between the robot's center of gravity and the wall, $G$ is the robot's gravity, $f_{y 1}$ and $f_{y 2}$ are frictional force on $y$ axis of two wheels respectively, $F_{N}$ is the adsorption force of the wheel, $N_{1}$ and $N_{2}$ are the support force of two wheels respectively. According to Fig. 2 , the robot's mechanical stability needs to meet the following conditions

$$
\left\{\begin{array}{l}
M_{y}=F_{N}\left(d_{1}+d_{2}\right)-N_{1}\left(d_{1}+\frac{d_{2}}{2}\right)-\frac{N_{2} d_{2}}{2}-G z_{1} \\
M_{x}=f_{y 1} x_{1}-f_{y 2} x_{2} \\
F_{y}=f_{y 1}+f_{y 2}-G
\end{array}\right.
$$


Where $M_{y}$ and $M_{x}$ are the robot's roll torque in $y$ axis and $x$ axis respectively, $F_{y}$ is the resultant force in $y$ axis direction. $M_{y} 、 M_{x}$ and $F_{y}$ should be 0. So, $F_{N} 、 f_{y 1}$ and $f_{y 2}$ can be calculated as follows

$$
\left\{\begin{array}{l}
F_{N}=\frac{N_{1}\left(d_{1}+d_{2} / 2\right)+N_{2}\left(d_{2} / 2\right)+G z_{1}}{d_{1}+d_{2}} \\
f_{y 1}=\frac{x_{2}}{x_{1}+x_{2}} G \\
f_{y 2}=\frac{x_{2}}{x_{1}+x_{2}} G
\end{array}\right.
$$

To prevent two wheels slip, $f_{y 1}$ and $f_{y 2}$ should be less than the wheel's maximum static friction force $f_{\max }$ which is been instead of sliding friction $\mu F_{N}$ ( $\mu$ is the wheel's friction coefficient). $N_{1}$ and $N_{2}$ should be more than 0 . So, the robot's stability condition can be given as follows

$$
F_{N} \geq \max \left(\frac{G x_{1}}{\mu\left(x_{1}+x_{2}\right)}, \frac{G x_{2}}{\mu\left(x_{1}+x_{2}\right)}, \frac{G z_{1}}{d_{1}+d_{2}}\right)
$$

Under the condition of transverse adsorption and motion, the robot's stress state is shown in Fig. 3.
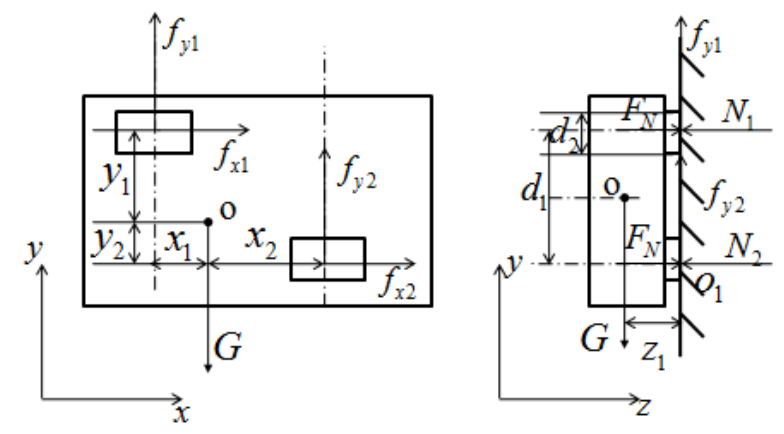

Fig. 3 Forces under the robot's lateral movement

In Fig. 3, $f_{x 1}$ and $f_{x 2}$ are the frictional force of two wheels respectively, which are provided by the drive motor torque $M_{1}$ and $M_{2}$. As shown in Fig. 3, the robot's stability conditions needs to meet the following

$$
\left\{\begin{array}{l}
M_{y}=F_{N}\left(d_{1}+d_{2}\right)-N_{1}\left(d_{1}+\frac{d_{2}}{2}\right)-\frac{N_{2} d_{2}}{2}-G z_{1} \\
M_{x}=f_{y 1} x_{1}+f_{x 1} y_{1}-f_{y 2} x_{2}-f_{x 2} y_{2} \\
F_{y}=f_{y 1}+f_{y 2}-G
\end{array}\right.
$$

(4)

$M_{1}=f_{x 1} r=M_{2}=f_{x 2} r$ ensures two wheels no side-slipping( $r$ is the wheel radius). So, we can obtain

$$
\left\{\begin{array}{l}
F_{N}=\frac{N_{1}\left(d_{1}+d_{2} / 2\right)+N_{2}\left(d_{2} / 2\right)+G z_{1}}{d_{1}+d_{2}} \\
f_{y 1}=\frac{x_{2}}{x_{1}+x_{2}} G+\frac{y_{2}-y_{1}}{x_{1}+x_{2}} \frac{M_{1}}{r} \\
f_{y 2}=\frac{x_{1}}{x_{1}+x_{2}} G+\frac{y_{1}-y_{2}}{x_{1}+x_{2}} \frac{M_{2}}{r}
\end{array}\right.
$$


Where, $y_{1}$ and $y_{2}$ are the distance between the front wheel and rear wheel and the robot's center of gravity in the $y$ direction.

To avoid two wheels slipping and flipping, the resultant force of $f_{x 1}$ and $f_{y 1}$, the resultant force of $f_{x 2}$ and $f_{y 2}$ should be less than $\mu F_{N}$, and $N_{1}$ and $N_{2}$ should be greater than 0 . So, the conditions of stability under the state of lateral movement can be obtained as follows

$$
F_{N} \geq \max \left(\frac{a}{\mu}, \frac{b}{\mu}, \frac{G z_{1}}{d_{1}+d_{2}}\right)
$$

Where $a=\sqrt{\left(\frac{G x_{1}}{x_{1}+x_{2}}+\frac{y_{1}-y_{2}}{x_{1}+x_{2}} \frac{M}{r}\right)^{2}+\left(\frac{M}{r}\right)^{2}}, \quad b=\sqrt{\left(\frac{G x_{2}}{x_{1}+x_{2}}+\frac{y_{2}-y_{1}}{x_{1}+x_{2}} \frac{M}{r}\right)^{2}+\left(\frac{M}{r}\right)^{2}}$.

When robot is being the vertical adsorption, the robot has the same force under the static state and moving state, which is shown in Fig. 4. The robot's mechanical stability conditions are

$$
\left\{\begin{array}{l}
M_{y}=\left(F_{N}-N_{1}\right)\left(x_{1}+x_{2}\right)-G z_{1} \\
M_{x}=f_{x 1} y_{1}-f_{x 2} y_{2}=0 \\
F_{y}=f_{x 1}+f_{x 2}-G \geq 0
\end{array}\right.
$$

In order to prevent two wheels to slip, $f_{y 1}$ and $f_{y 2}$ should be less than $f_{\max }$ displaced by $\mu F_{N}$. In addition, two wheels' supporting force should be greater than 0 for avoiding the robot to flip. So, the robot's motion stability condition can be obtained as follows
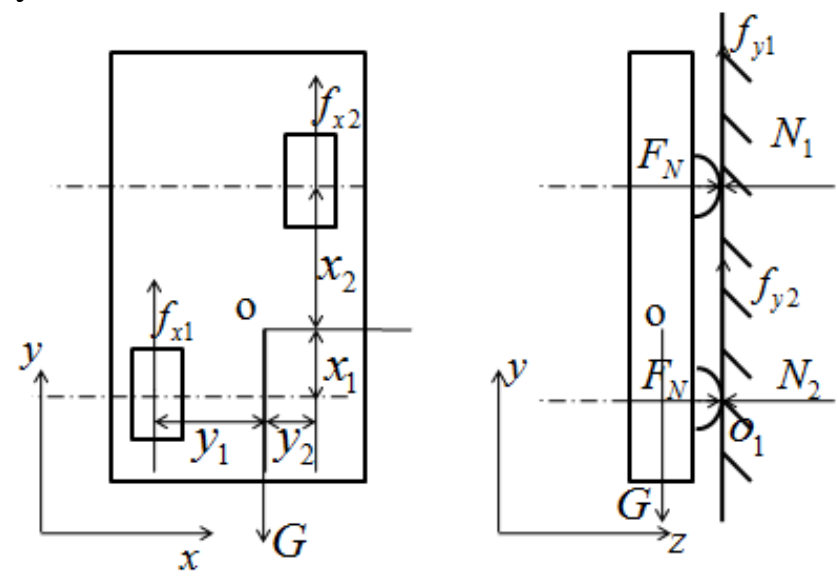

Fig. 4 Forces under the robot's vertical adsorption condition

(8)

$$
F_{N} \geq \max \left(\frac{G z_{1}}{x_{1}+x_{2}}, \frac{G y_{1}}{\mu\left(y_{1}+y_{2}\right)}, \frac{G y_{2}}{\mu\left(y_{1}+y_{2}\right)}\right)
$$

Equation (3), Equation (6) and Equation (8) are the wall-climbing robot's stabilization conditions of adsorbing wall under all states.

\section{Simulation Analysis}

This paper sets all parameters including the robot body, load and kinematic according to the mechanical model and stability conditions established in the previous section. The robot's virtual prototype model is built by Solidworks. And then the model is imported into ADAMS to simulate the stress and motion of the climbing robot on the wall. For the limitations of coverage, this paper only simulates one form motion which the robot climb along the wall straight upward with a certain velocity. We analyze the robot's trend of away from the wall by data of the distance between two wheels and the wall. 
Fig. 5 shows the change curve of the distance between two wheels and the wall. Fig. 5 (a) and (b) show the distance curve of the left wheel and the right wheel respectively. It can be seen that the two wheels have a small displacement at the beginning of the movement. In the initial stage of the movement, driving wheel contact with the wall tightly under the action of magnetic force. And then, the distance is maintained a constant value. Simulation results show two wheels has never left the wall throughout the robot motion, and the robot can overcome the action of gravity and keep linear motion.

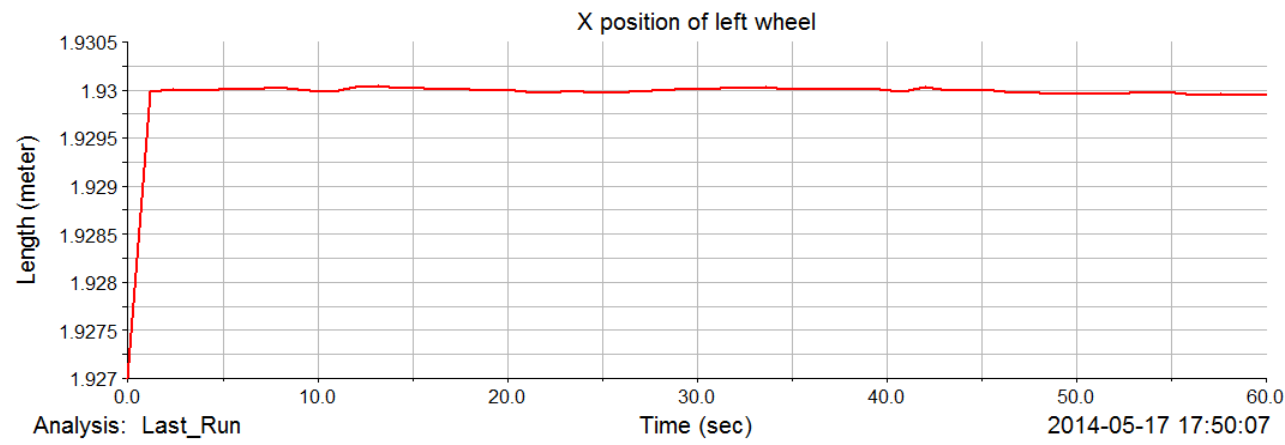

(a) The curve of left wheel

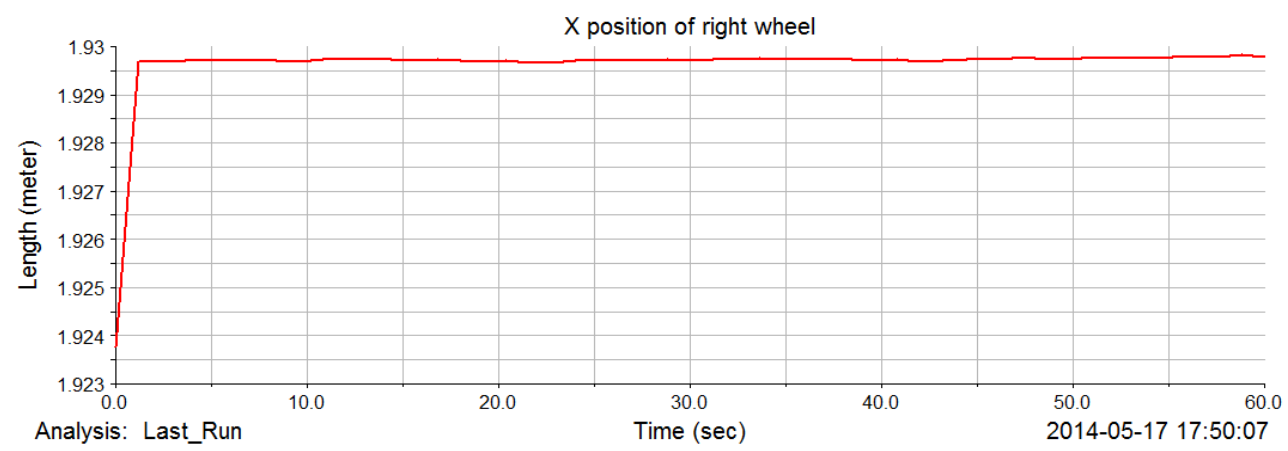

(b) The curve right wheel

Fig. 5 The change curve of distance between the robot and the wall

\section{Conclusion}

This paper designs a four-wheel mobile structure based on permanent magnetic adsorption for wall-climbing robot, which is constituted by two permanent magnet driving wheel and two universal auxiliary wheels.

This paper establishes the stability conditions under all robot's motion and static state by mechanics analyzing.

Some simulation experiments verify the correctness of the mechanics model and the stability condition. These conclusions will provide references for the design of wall-climbing robot.

\section{Acknowledgement}

In this paper, the research was sponsored by the National Nature Science Foundation of China (Project No. 61373110 and Project No.61272196) and Wuhan Science and Technology Research Projects (Project No. 2014010101010005).

\section{Reference}

[1] R X Zhang, J C Latombe. Capuchin: A Free-Climbing Robot [J]. International Journal of Advanced Robotic Systems 2013,10(194):1-18.

[2] A Subramanyam, Y Mallikarjuna, S Suneel Et al. Design and Development of a Climbing Robot 
for Several Applications [J]. International Journal of Advanced Computer Technology,2013.3(3):15-23.

[3] R D Dethe1, S B Jaju. Developments in Wall Climbing Robots: A Review [J]. International Journal of Engineering Research and General Science,2014,2(3):33-42.

[4] Y L Fu, Z H Li. Researching headway of wall-climbing robots [J]. Journal of Machine Design.2008,25(4):1-5.

[5] M Eich , T Vogele. Design And Control Of A Lightweight Magnetic Climbing Robot For Vessel Inspection [C]. IEEE 19th Mediterranean Conference on Control and Automation Aquis Corfu Holiaday Palace, Corfu.Greece, 2011,June, 20-23:1200-1205.

[6] J D Wang, H Kong, Q D Chen Et al. The Walk-adsorption Mechanism Design of a New Tank-Wall-Climbing Inspection Robot [J]. Machinery Design \& Manufacture 2013.(1)26-28. 